\title{
FLUID DAMPING OF AN ELECTROSTATIC ACTUATOR FOR OPTICAL SWITCHING APPLICATIONS
}

\author{
Kevin Y. Yasumura, John D. Grade, and Hal Jerman \\ Micromachining Development Group \\ io $\lambda$ on Inc. \\ 1870 Lundy Avenue \\ San Jose, CA 95131-1826
}

\begin{abstract}
A $1 \times 4$ optical switch has been fabricated using a micromachined rotary actuator and commercially available miniature optics. This switch features an electrostatic actuator and a separately fabricated out-of-plane mirror. An important characteristic of such a device is its switching speed. Improved switching time can be obtained by damping the fundamental mode of motion to reduce the effect of ringing when the switch output is changed to a different fiber. In addition, damping the motor motion will improve the switch's immunity to externally applied accelerations. One means to passively damp the actuator modes of motion is to use a damping fluid with a viscosity greater than air (190 uP). Switching times of $\sim 1 \mathrm{msec}$ have been obtained for a fluid filled switch with less than $0.5 \mathrm{~dB}$ insertion loss.
\end{abstract}

\section{INTRODUCTION}

There are a number of applications that require low port count optical switches. These applications include adding protection and restoration capabilities to an optical network, optical component testing, and network monitoring. Using an optical switch, a failed fiber or transmitter link can be quickly bypassed until it can be repaired. In a component testing application, low port count optical switches can be used to test a series of optical components using the same set of test hardware. This can significantly reduce equipment cost and increase testing throughput. Real-time network monitoring and diagnostic applications are also possible through the use of optical switches. All of these applications can be accomplished using low port count optical switches that can be scaled to higher port numbers if needed.

In long-haul optical networks, all-optical switching provides a means to reduce overall system cost by reducing the number of times light must be converted to an electrical signal and again to an optical signal (OEO conversion). This process is expensive, requiring both a receiver and a transmitter to resend the optical signal. Optical transparency is becoming increasingly important for next generation network system designers as the percentage of node pass-through traffic increases. All-optical switches also have the advantage of being transmission protocol and bit-rate independent.

One version of a low port count optical switch is the $1 \times 4$ switch shown in Fig. 1. The switch consists of a micromachined rotary electrostatic actuator, a reflective mirror, a ceramic substrate, a GRIN lens, and an array of optical fibers. This separation of components allows for best-of-class component selection without concern for process compatibility. In addition, such a process allows for improved yield and flexibility in design. Shown in Fig. 1 is a 5 fiber array: 1 fiber is the input fiber (center fiber) and the other four fibers are the switch output fibers. The GRIN lens collimates the input beam from the fiber array and focuses it onto the mirror which is placed so that the axis of rotation is at the focal point of the lens. The light then reflects off of the mirror, passes back through the lens, and is refocused onto one of the output fibers. The actuator selects which output is used by rotating the mirror such that the input beam is reflected toward the selected output fiber. The entire switch package is approximately $1 " \times 1 " \times 0.5$ " including the necessary drive and control electronics.

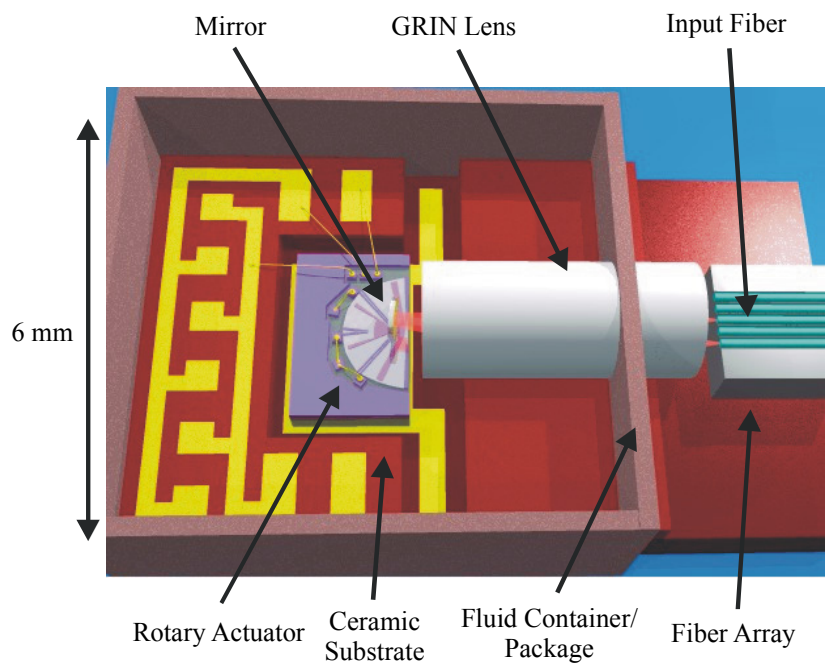

Figure 1. Diagram of a $1 \times 4$ optical switch using a micromachined rotary actuator, a micromachined mirror, a GRIN lens, and a fiber array consisting of the input optical fiber and four output fibers. The switch is fabricated on a ceramic substrate and placed within a small 8 pin DIL package.

Figure 2 shows a SEM photograph of a rotary actuator with a $12^{\circ}$ total rotation range [1]. An early, undamped switch of this type was made with less than $0.5 \mathrm{~dB}$ insertion loss and with $<5$ msec switching times between channels [2].

The resonance frequency of the switch actuator as well as its quality factor, $Q$, limit the optical switching time. In an open-loop system, high $Q$ will produce ringing during a switching event, and it is therefore useful to reduce the $Q$ of the fundamental mode of oscillation. Because device size and cost are important considerations for a commercial product, a reliable yet inexpensive means to damp the motor motion was sought. This work focuses on reduction in quality factor for such a micromachined actuator using a passive, fluid-filled damping approach to improve switching time performance.

\section{OPTICAL SWITCH FABRICATION AND DESIGN}

The process for fabricating the micromachined actuator has been described in detail in previous work [3]. First, a substrate 


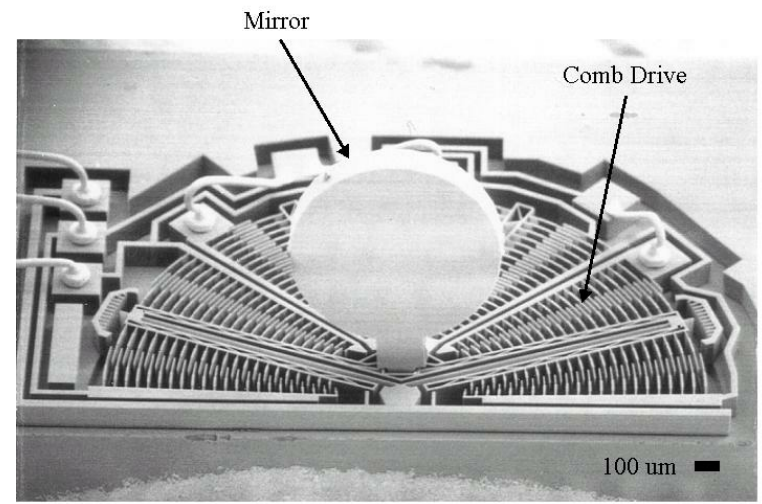

Figure 2. SEM photograph of a switch actuator and its reflective mirror. The rotary switch motor has a total rotation range of 12 degrees with a maximum applied voltage of $150 \mathrm{~V}$. The actuator die size is $2.8 \times 1.8 \mathrm{~mm}$. Two sets of combs rotate the actuator about an axis normal to the die surface (yaw). Applying a voltage to the electrically-isolated silicon substrate allows the mirror to be tilted upwards (pitch) to a maximum of 0.2 degrees with an applied voltage of $80 \mathrm{~V}$.

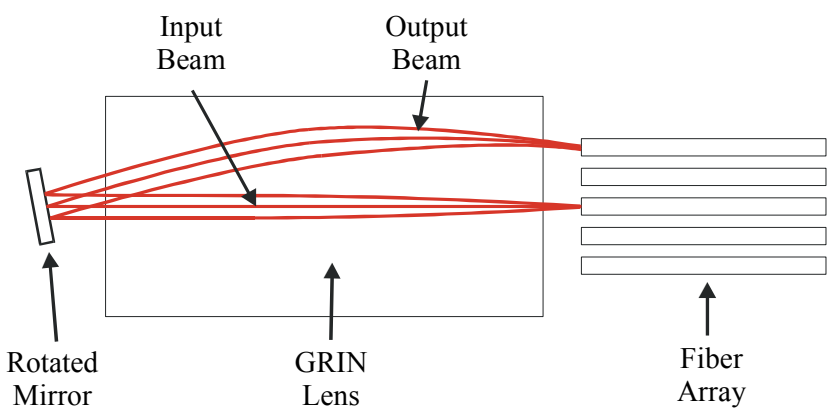

Figure 3. Diagram of the optical switch in operation. In this figure, the MEMS actuator has been rotated to switch the input light into the upper-most output fiber. The MEMS rotator can be instructed to quickly switch the input light to any of the output fibers.

silicon wafer is patterned with a 15-um deep cavity. The micromachined actuator will be fabricated above these cavities in the substrate wafer. Next, the device wafer is fusion bonded to the substrate wafer. The wafer stack is then ground and polished to produce the desired total motor thickness (typically 85-um thick). Contact pad metalization is then deposited and patterned. Finally, the motor features are defined using a DRIE process. Figure 2 shows a fabricated motor with its mirror attached. The paddleshaped mirror is a gold-coated silicon plate with an attachment peg that is inserted into the motor. This mirror can be fabricated using the same process as the actuators. Passive placement of the mirror onto the rotary micromachined actuator using vision-guided robotics can routinely produce $<0.1$ degrees of out-of-plane tilt.

In order to drive the actuator, a maximum voltage of $150 \mathrm{~V}$ is applied to one of two sets of electrostatic combs. Although the actuator requires a fairly high voltage to move to full angular rotation, the current draw is very small (pA) producing a low power device $(\mathrm{nW})$. One set of combs causes the motor to rotate in the clockwise direction while the opposite set of combs will make the motor rotate in the counter-clockwise direction. The rotation of the actuator allows the switch to steer the incoming light into one of the output fibers. The actuator shown in Fig. 2 has a typical fundamental resonance frequency of $700 \mathrm{~Hz}$ allowing it to make rapid transitions between the various output states.

A diagram of the switching process is shown in Fig. 3. In this figure, the micromachined actuator has been rotated such that the incident light coming into the switch from the input fiber is reflected toward the top output fiber. The output fiber can be selected by simply moving the high-speed actuator to a new position. Accurate targeting of the output fiber is crucial to limit the amount of power loss in the switch. A radial beam misalignment of only $1 \mathrm{um}$ from the center of the output fiber core will reduce the coupled power by $0.22 \mathrm{~dB}$ assuming purely gaussian beam coupling.

This issue becomes very important if you consider that the fiber cores in the fiber array are not perfectly collinear. The slight misalignment in each fiber is due to core centration error in the fiber itself and variability in the fabrication of the fiber array. Typically, about a $1 \mathrm{um}$ height variation is found in a given fiber array. Figure 4 shows a plot of fiber core center position as a function of height along the y-axis (normal to the plane of the switch ceramic) for a typical array. This figure shows that an actuator that can only rotate (i.e., translate the beam in the $\mathrm{x}$ direction) will not be able to optimally couple light into all of the output fibers. A means to deal with this is to create an actuator that can rotate in the in-plane direction but can also tilt in the outof-plane direction [4]. The micromachined rotary actuator shown in Fig. 2 features this out-of-plane tilting capability. By applying a voltage to the electrically-isolated substrate wafer, the actuator can be attracted toward the substrate. For a typical 15-um deep etch cavity, this will produce a maximum of 0.2 degrees of out-of-plane tilting. This is more than enough motion to allow the actuator to efficiently couple light into all of the output fibers of the fiber array.

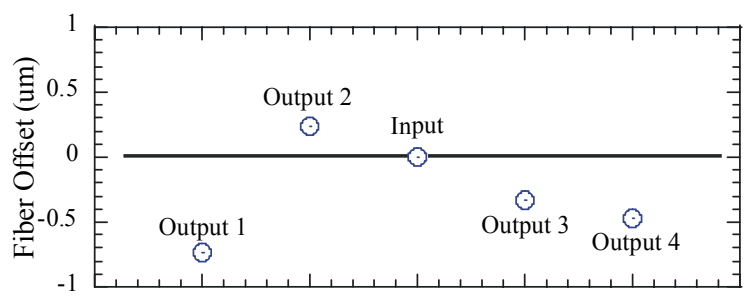

Figure 4. Plot of the fiber core positions for a typical fiber array. In each fiber array the fiber cores are not perfectly aligned in the $y$-axis direction potentially causing additional coupling loss. Typical fiber array height variation is $1 \mathrm{um}$.

Another issue that is resolved by using an actuator that can rotate about two axes is fiber-crossing during switching events. Instead of simply sweeping across all of the intermediary fibers, such an actuator can raise the beam above the fiber cores, rotate to the desired output fiber, and then tilt the beam back into position for the output fiber. Figure 5 shows a diagram of this type of switching event. By following the non-crossing switching path, the fiber crosstalk can be reduced significantly.

Because of the DRIE process used to fabricate the rotary actuators, high capacitance structures are created. The change in capacitance as the actuator rotates is $>1 \mathrm{pF}$. This allows for the use of off-chip capacitance circuitry that can be used to infer the position of the rotary actuator during operation. When the switch is fabricated, all of the initial components can be placed passively. The fiber array is the last component to be placed onto the switch. During the placement process, the fiber positions are optimized for coupled power. These optimal coupling voltages are then stored in 
the control electronics. Future switching events then use these programmed voltages values together with the capacitance circuitry to provide a degree of closed-loop control for the actuator motion. The out-of-plane actuator motion is open-loop.

The long-term reliability of micromachined devices is often of concern. For the high-aspect ratio MEMS devices used in Iolon Corporation's products, however, excellent motor reliability has been demonstrated. A set of DRIE actuators, similar to the type shown in Fig. 2 has been actuated over 60 billion times with no measurable change in performance.

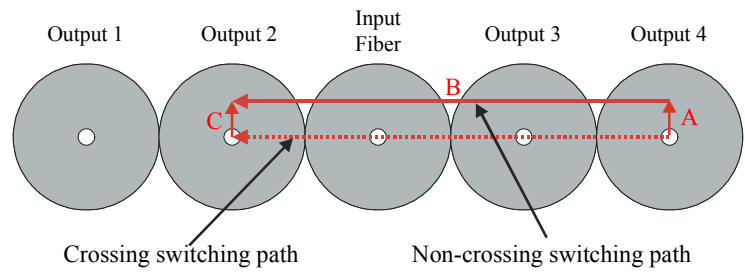

Figure 5. In addition to being able to deal with the fiber core alignment issue, it is desirable to have a non-crossing switch: i.e., when switching between the different output fibers, light is not inadvertently sent into one of the intermediary fibers. This is shown in the diagram above. In the crossing switching path, light will be sent into output fiber 3 before the beam reaches output fiber 2. This is resolved by having an actuator that can first raise the beam above the fiber core positions (path A), cross over to the desired output fiber (path $B$ ), and then tilt the beam down and into the target output fiber core (path $C$ ). Note: switching paths are not drawn to scale.

\section{SWITCH PERFORMANCE \& FLUID DAMPING}

For MEMS devices fabricated from materials such as silicon, silicon carbide, silicon nitride, and polysilicon, the intrinsic material damping can be very low. $Q$ 's greater than $10^{3}-10^{4}$ can be obtained for silicon micromachined structures operated in vacuum [5,6]. Such high $Q$ 's are of value to improve measurement resolution if used as a resonant sensor. In a switching application, however, high $Q$ produces residual ringing of the device when it is switched between channels. A reduction of $Q$ preferably below 1 is therefore desired. For MEMS devices operated in atmosphere, air damping is usually the dominant damping mechanism $[\mathbf{6}, 7, \mathbf{8 , 9}]$.

The fundamental resonance of the switch actuator shown in Fig. 2 has a measured $Q$ of 20 in air. When compared with intrinsic $Q$ values of single-crystal silicon structures, this is already a large amount of damping. Additional damping, however, is desired in order to reduce the $Q$ further. Because the gap between moving elements is limited by fabrication design rules, additional gas damping via design modifications is difficult to obtain. Another means to increase damping is to fill the actuator package with a damping fluid with a viscosity greater than air (190 uP) $[\mathbf{1 0 , 1 1}]$. There are a large number of possible fluids that could be used. Gases such as Ne (315 uP viscosity) can produce small amounts of additional damping. An ideal damping fluid would have a viscosity sufficient to bring the $Q$ close to unity but low enough to allow high-speed operation.

The damping fluid must also be nonconductive to allow proper operation of the electrostatic comb drive. Additionally, a liquid will have a dielectric constant higher than air. This will benefit the motor's motion because the force between the actuator combs is proportional to the dielectric constant of the material between the combs. Polar fluids typically have higher dielectric constants.

Two different fluids were tested: a proprietary low-viscosity oil (Cargille 4501) and diethylbenzene (DEB). If the fluid damping is modeled using Stokes flow damping, the $Q$ can be expressed as

$$
Q=\frac{g}{\mu A} \sqrt{k m},
$$

where $\mu$ is the fluid viscosity, $A$ is the comb overlap area, $k$ is the motor's suspension stiffness, $m$ is the moving mass, and $g$ is either the boundary layer thickness or the gap between the moving elements [8]. The low-viscosity oil has a viscosity of $1.4 \mathrm{cP}$ while diethylbenzene is $0.6 \mathrm{cP}$ (measured using a falling ball viscometer). With the Stokes model, the predicted $Q$ 's are 0.25 for the oil and 0.60 for diethylbenzene. Figure 6 shows measured frequency responses in air, in oil, in diethylbenzene, and again in air after the fluid fill tests. The measured and predicted quality factors differ by less than $15 \%$. The pre- and post-air tests produced identical damping results.

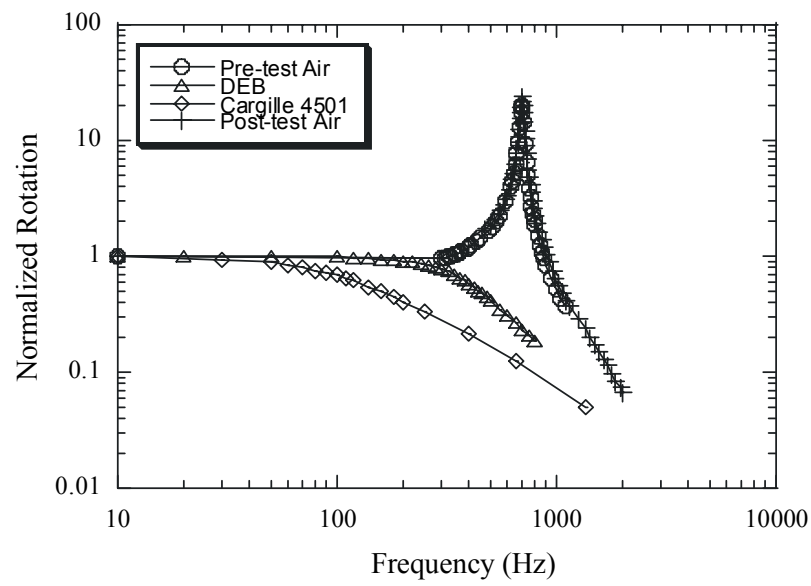

Figure 6. Frequency response plot of the switch actuator. The motor was measured in air, in a low viscosity oil (Cargille 4501), in diethylbenzene (DEB), and again in air after removal of the fluid damping materials. The introduction of the fluid fill materials has reduced the fundamental $Q$ below 1 in both cases. The motor returned to its initial damping value after removal of the fluid damping materials.

Figure 7 shows a fiber-switching event with an applied step voltage. Data for switching both in air and in a damping fluid is shown. In air, considerable ringing is observed in the output fiber after the switching event. The fundamental, in-plane $Q$ of the actuator $(20)$ produces ringing as the motor settles into its new alignment position. A total switching time of $>50 \mathrm{msec}$ was observed under these conditions. By reducing the fundamental $Q$ of the actuator, this ringing response can be removed. Fluid damping the rotary actuator produces a significant improvement in the fiber switching time.

Figure 8 shows a zoomed view of the fiber-switching event in a fluid environment. Diethylbenzene damped the motor's fundamental $Q$ to below 1. This fluid damping enabled switching times of $\sim 1 \mathrm{msec}$. The drop in power after first light is caused by slight overshoot of the actuator as it switches to the new output fiber.

A secondary benefit of using a fluid-filled damping scheme is the enhancement of the electrostatic force caused by the increased dielectric constant of the fluid material. Because the force created 


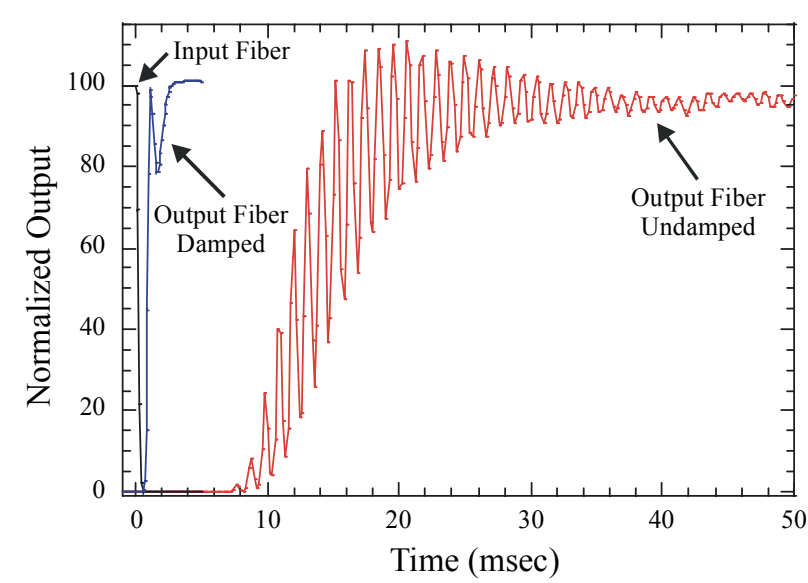

Figure 7. Fiber switching in air and in a damping fluid (DEB) with an applied step voltage. The ringing observed in air is caused by this step change in drive voltage. The $Q$ of the actuator (20) increases the decay time of the post-transition oscillation, producing switching times $>50 \mathrm{msec}$. One means to reduce this ringing effect is to reduce the actuator quality factor. After application of the damping fluid, the switching time (and amount of ringing) has been reduced significantly.

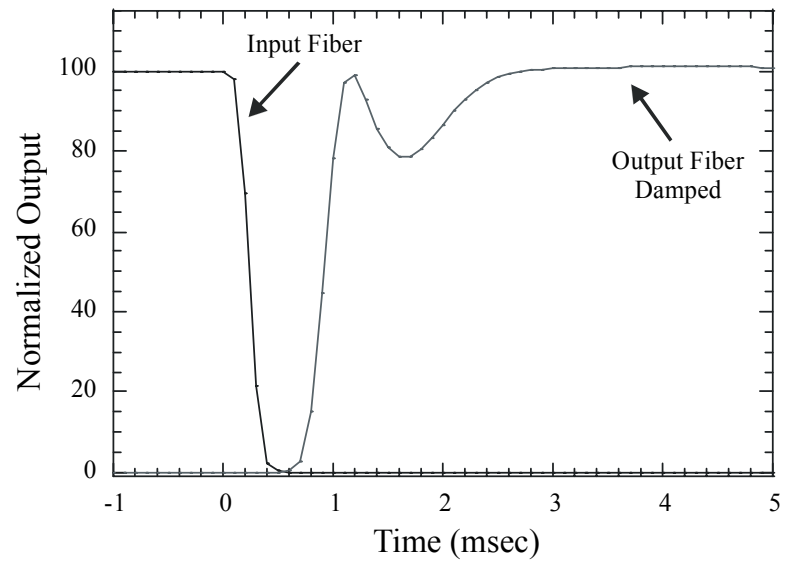

Figure 8. Fiber switching in diethylbenzene (DEB). The ringing observed in the output fiber when switching in air is dramatically reduced by damping the actuator's fundamental oscillation mode to have a $Q$ less than 1 . Note the reduced time scale: the damped switching time is just over 1 msec to first light. The $1 \mathrm{~dB}$ power drop is caused by slight rotational overshoot of the switch actuator.

by the comb drive is proportional to $\varepsilon V^{2}$, where $\varepsilon$ is the dielectric constant of the fluid-fill material and $V$ is the applied voltage, the amount of voltage required to drive the actuator to full deflection is reduced. In air, the voltage required to reach full in-plane angular rotation is $150 \mathrm{~V}$. This maximum voltage is reduced when the switch is filled with a fluid damping material. For DEB, the voltage reduction indicated a dielectric constant of 2.44 while for Cargille 4501 the dielectric constant was 3.45. This is a considerable increase in the electrostatic force. Other types of actuator systems could potentially benefit from using such a forceenhancement scheme when actuator size or voltages are limited by experimental or design considerations.

Additional issues with this concept might include possible fluid degradation over time. This is especially important if high optical powers are to be switched using this device. Additionally, the effect of the fluid on the optical surfaces and coatings needs to be investigated to ensure reliable switch operation over the lifetime of the device. Finally, to limit the switch insertion loss, attention to the optical absorption spectrum of the selected fluid material also needs to be investigated.

\section{CONCLUSIONS}

A novel method to passively damp an all-optical electrostatic switch actuator has been demonstrated. $Q$ 's $<1$ have been obtained while simultaneously improving the switching time performance. Switching times of $\sim 1 \mathrm{msec}$ have been obtained for a packaged $1 \times 4$ microswitch device.

This passive, fluid-based damping technique offers advantages not only to the measured switching time but it should also improve the vibration immunity of the device. The use of nonconductive, high dielectric constant materials also produces an enhancement to the electrostatic force used to actuate the MEMS device. This results in a reduction in the voltage required to move the mirror rotator to full angular deflection.

\section{ACKNOWLEDGEMENTS}

The authors would like to thank Al Fennema, Steve Hrinya, Kathy Jackson, Howard Lee, Pei-Fei Li, and Chris Tan at Iolon, Inc. and Sam Wong at TRW Lucas Novasensor for their assistance with fabrication, assembly, and testing.

\section{REFERENCES}

1. J.H. Jerman and J.D. Grade, US Patent 6,329,737 B1, "Rotary Electrostatic Microactuator", Dec 11, 2001.

2. J.D. Grade and J.H. Jerman, "MEMS Electrostatic Actuators for Optical Switching Applications", Optical Fiber Conference, WX2$1,2000$.

3. J.D. Grade, H. Jerman, and T.W. Kenny, "A large-deflection electrostatic actuator for optical switching applications", Proc. 2000 Solid-State Sensor and Actuator Workshop, Hilton Head, SC, pp. 97-100.

4. J.H. Jerman and J.D. Grade, US Patent 6,333,584 B1, "Tiltable Electrostatic Actuator and Method for Using Same", Dec 25, 2001. 5. R.E. Mihailovich and N.C. MacDonald, "Dissipation measurements in vacuum-operated single-crystal silicon microresonators", Sens. Actuators, vol. 50, pp. 199-207, 1995.

6. K.Y. Yasumura, et al., "Quality factors in micron- and submicron-thick cantilevers", Jour. Microelec. Mech. Sys., vol. 9, no. 1, pp. 117-125, 2000.

7. W.C. Tang, et al., "Laterally driven polysilicon resonant microstructures", Proc. IEEE Workshop on Micro. Electro. Mech. Syst., Salt Lake City, UT, U.S.A., Feb. 1989, pp. 53-59.

8. Y.H. Cho, et al., "Viscous Energy Dissipation in Laterally Oscillating Planar Microstructures: A Theoretical and Experimental Study", Proc. IEEE Conf. Of Micro. Elect. Mech. Systems, Ft. Lauderdale, FL, Feb 1993.

9. F.R. Blom, et al., "Dependence of the quality factor of micromachined silicon beam resonators on pressure and geometry", J. Vac. Sci. Technol., B10, 1, 1992, pp. 19-26.

10. L. Roylance and J. Angell, "A batch fabricated silicon accelerometer”, IEEE Trans. on Elec. Dev., vol. ED-26, no. 12, pp. 1911-1917, Dec 1979.

11. J.D. Grade, J.H. Jerman, and J.D. Drake, PCT International Publication number W0 01/95468, "Micromechanical Device With Damped Microactuator”, Dec 13, 2001. 